\title{
Change in lumbar lordosis during prone lying knee flexion test in subjects with and without low back pain
}

\author{
Amir M Arab ${ }^{1 *}$, Ailin Talimkhani ${ }^{2}$, Noureddin Karimi ${ }^{2}$ and Fetemeh Ehsani ${ }^{2}$
}

\begin{abstract}
Background: Prone lying knee flexion (PLKF) is one of the clinical tests used for assessment of the lumbo-pelvic movement pattern. Considerable increase in lumbar lordosis during this test has been considered as impairment of movement patterns in lumbar-pelvic region. However, no study has directly evaluated the change in lordosis during active PLKF test in subjects with low back pain (LBP). The purpose of this study was to investigate the change of lumbar lordosis in PLKF test in subjects with and without LBP.
\end{abstract}

Methods: A convenience sample of 80 subjects participated in the study. Subjects were categorized into two groups: those with chronic non-specific LBP ( $N=40$, mean age: $40.84 \pm 17.59)$ and with no history of LBP ( $N=40$, mean age: $23.57 \pm 10.61)$. Lumbar lordosis was measured with flexible ruler, first in prone position and then on active PKF test in both subjects with and without LBP. Data was analyzed by using statistical methods such as, independent t-test and paired t-test.

Results: There were statistically significant differences in lumbar lordosis between prone position and after active PLKF in both subjects with and without LBP ( $\mathrm{P}<0.0001)$. The amount of change in lordosis during PLKF test was not significant between the two groups $(P=0.65)$. However these changes were greater among patients with LBP.

Conclusion: Increase in lumbar lordosis during this test may be due to excessive flexibility of movement of the lumbar spine in the direction of extension and abnormal movement patterns in the individuals with LBP.

Keywords: Low back pain, Lumbar lordosis, Movement pattern, Prone knee flexion, Flexible ruler

\section{Background}

Low back pain (LBP) is a world-wide health problem and the most common and costly musculoskeletal disorder in the today's societies $[1,2]$. The prevalence of LBP is estimated to be between $10 \%$ and $80 \%$ depending on the population [3,4]. Despite its high prevalence and detrimental effects on subjects' activities, the exact causes of mechanical LBP have not yet been fully understood. However, during the past decades the approach in evaluation and management of LBP has been changed from strengthening or stretching of the lumbo-pelvic muscles toward modification of the motor system and movement pattern [5].

\footnotetext{
*Correspondence: arabloo_masoud@hotmail.com

'Department of Physical Therapy, University of Social Welfare and

Rehabilitation Sciences, Velenjak, Tehran, Iran

Full list of author information is available at the end of the article
}

A balanced motor system is obtained from coordinated activity of synergist and antagonist muscles. Normal functioning of the trunk depends not only on passive joint mobility, but also on normal muscular activity and central nervous system regulation. Muscles produce and control the movement and stabilize the spine, protecting if from excessive load during functional activities [6,7].

With regard to this point of view, repetitive movements and long-term faulty postures and movements can change muscle tissue characteristics and can lead to muscle dysfunction, altered movement pattern, pain and finally movement disorders [5]. Hence, the main emphasis has been recently placed on assessment of the altered movement pattern in patients with musculoskeletal pain and disorders such as LBP and on the important of achieving normal pattern of the movement for the prevention and treatment of LBP [6-11]. 
Several studies have demonstrated that LBP is associated with muscle imbalance and altered activation pattern of the lumbo-pelvic muscles during different tasks [12-15]. Some clinical tests have been used to assess the altered movement pattern in subjects with musculoskeletal disorders. Prone lying knee flexion (PLKF) is an accepted test for assessment and treatment of the lumbo-pelvic movement patterns [5]. In this test, a patient lays prone and actively flexes his or her dominant knee as far as possible. Muscle imbalance and altered activation of the lumbopelvic muscles has been reported during PLKF test in patients with chronic LBP [5]. Excessive anterior pelvic tilt, lumbar rotation, lumbar hyperextension, increased lumbar lordosis and decreased knee flexion during the PLKF has been considered as abnormal movement patterns during PLKF [5]. Coordination between muscles in the lumbopelvic region is thought to balance the position of the pelvis in normal posture and during the lower limb or trunk movement. It has been assumed that during PLKF, lack of sufficient stiffness in the abdominal and anterior supporting structures of the lumbar spine produces anterior tilt in the pelvic and increased lumbar lordosis specially in person with lower cross syndrome [5].

However, to our knowledge, no study has investigated the change in lumbar lordosis during PLKF in patients with chronic LBP. The purpose of this study was to investigate the change in the degree of lumbar lordosis during PLKF in subjects with and without chronic LBP and to determine if this change varies between two groups.

\section{Methods}

\section{Subjects}

The quasi-experimental study design with repeated measurements was used to investigate the lumbar lordosis changes during PLKF in two groups: subjects with chronic non-specific LBP $(\mathrm{N}=40$, average age: 40.84 [SD = 17.59] years old, average height: $165.0[\mathrm{SD}=9.0] \mathrm{cm}$, average weight: $70.31[\mathrm{SD}=16.06] \mathrm{kg}$, body mass index $(\mathrm{BMI})$ : $25.55[\mathrm{SD}=3.99] \mathrm{kg} / \mathrm{m}^{2}$ ) and subjects with no history of LBP $(\mathrm{N}=40$, average age: $23.57[\mathrm{SD}=10.61]$ years old, average height: 162.0 [SD $=7.0] \mathrm{cm}$, average weight: 55.62 [SD = 6.55] kg, BMI: 21.05 [SD = 2.26] kg/m²).

Power analysis was used to determine the sample size for test. Type I error $(\alpha)$ was set at 0.05 and power of the test was 0.80 . Considering this, the calculated sample size showed that sample size in this study was appropriate to test the hypothesis and the results derived from the study are meaningful.

The subject population in this study was a sample of convenience. The LBP patients were referred by orthopedic specialist and physiotherapy clinics. The patients were included if they had a history of non-specific LBP for more than six weeks duration before the study date. They were also included if had intermittent (on and off) LBP with at least three previous episodes each lasting more than one week, during the year before the study [16].

The control group was evaluated and found to have no complaint of any pain or dysfunction in their low back, pelvis, thoracic and lower extremities. The healthy subjects were recruited from the university students.

The exclusion criteria in both groups were pregnancy, history of dyspnea, history of hip pain, dislocation or fracture, history of lumbar spine surgeries, history of anterior knee ligament injury or rupture, history of anterior knee pain, inability to perform active PLKF without pain, history of lower extremity injury in the past 3 months, shortness of hip flexors, positive neurological symptoms and cardiopulmonary disorders. Each eligible subject was enrolled after signing an informed consent form approved by the human subjects committee at the University of Social Welfare and Rehabilitation Sciences. Ethical approval for this study was granted from the internal ethics committee at the University of Social Welfare and Rehabilitation Sciences (Date: 2013.03.09).

\section{Procedures}

The subject was on the examining table in the prone position. The lumbar lordosis was measured first in prone position. Then the subject was asked to perform knee flexion (PLKF) and then the lumbar lordosis was measured after PLKF test in both subjects with and without LBP. The dominant leg was chosen for investigation.

\section{Measuring lumbar lordosis}

A standard flexible ruler was used to measure the degree of lumbar lordosis in prone position before and after active knee flexion (Figure 1). For this purpose, the subject's position was prone lying on a treatment table with the arms along the sides and head face was down. The base of sacrum and spinous process of L1 was located by palpation and marked with removable stickers.

A standard flexible ruler was fitted on subject's lumbar curve, over the lumbar spinous processes of L1 - S1. The curve of the flexible ruler, resembling the size of subject's lumbar curvature, was graphed on a paper, noting where the two reference points for L1 and S1 were located. The method explained by others was used to quantify the degree of lumbar lordosis [16-20].

Two points on the curve, representing $\mathrm{L} 1$ and $\mathrm{S} 1$, were connected by a line $(\mathrm{L})$. A perpendicular line $(\mathrm{H})$, representing the height of the lumbar curve, bisected line L. The length of each line was calculated in millimeters, and the values were used in the following formula to calculate the degree of lumbar lordosis.

$$
\theta=4[\operatorname{Arctan}(2 \mathrm{H} / \mathrm{L})]
$$

A very high correlation $(r=0.92)$ has been found between degrees of lumbar lordosis measured by a flexible 

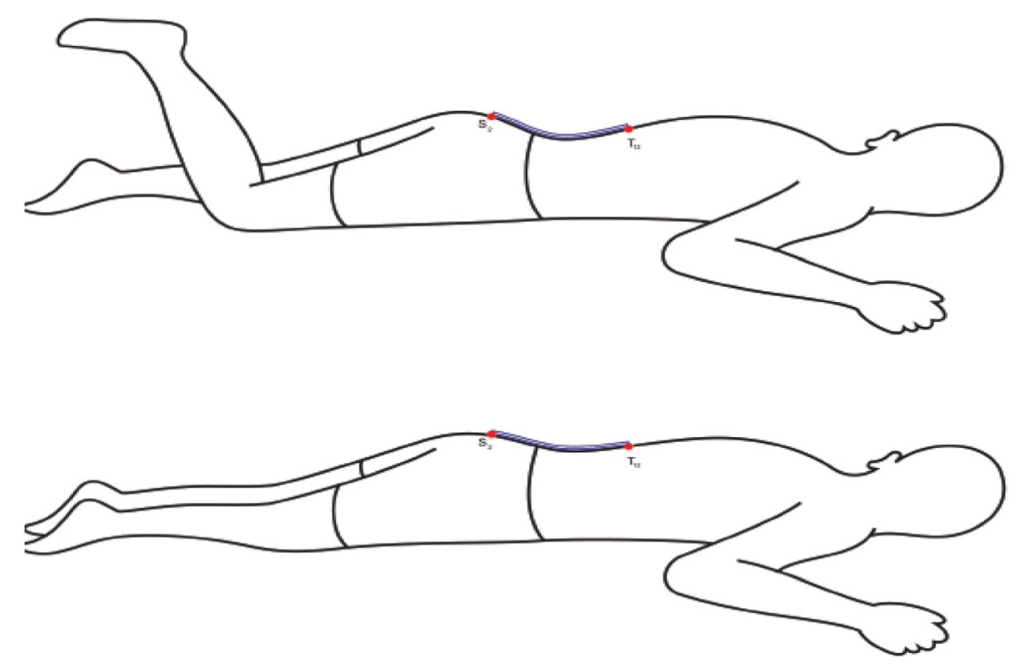

Figure 1 Measurement of lumbar lordosis with flexible ruler in prone position and active PLKF.

ruler and from lumbar X-rays [21-23]. The reliability of flexible curve for measurement of lumbar lordosis has been previously established [24].

\section{Data analysis}

Statistical analysis was performed using SPSS version 16.0.

A paired $t$-test was used to demonstrate changes in lumbar lordosis before and after PLKF test in both subjects with and without LBP.

An independent $t$-test was used to compare changes in lumbar lordosis during PLKF between subjects with and without LBP and also to compare demographic data between subjects with and without LBP. Statistical significance was attributed to $\mathrm{P}$ value less than 0.05 .

\section{Ethical approval}

This research was reviewed and was approved by the Human Subject Committee at University of Social Welfare and Rehabilitation Sciences.

\section{Results}

The demographic data for the subjects are presented in Table 1. No statistical significance was found in the height between groups. However, there was a statistically

Table 1 Demographic data of the subjects in each group

\begin{tabular}{lll}
\hline Variables & With no LBP $(\mathbf{n}=\mathbf{4 0})$ & With LBP $(\mathbf{n}=\mathbf{4 0})$ \\
\hline Age (years) & $23.57(10.61)$ & $40.84(17.59)$ \\
Weight $(\mathrm{kg})$ & $55.62(6.55)$ & $70.31(16.06)$ \\
Height $(\mathrm{cm})$ & $162.0(7.0)$ & $165.0(9.0)$ \\
BMl $\left(\mathrm{kg} / \mathrm{m}^{2}\right)$ & $21.05(2.26)$ & $25.55(3.99)$ \\
\hline
\end{tabular}

Continuous data: Mean (Standard Deviation). $\mathrm{LBP}=$ Low Back Pain, BMI = Body Mass Index. significant difference in subjects' age, weight and BMI between the two groups $(P=0.000)$.

There was no significant difference in lumbar lordosis at the baseline in prone relaxed position between two groups ( $\mathrm{P}=0.21$, \%95 CI: $1.77-7.44)$. There was a statistically significant difference in lumbar lordosis between prone position and after PLKF in subjects without LBP $(\mathrm{P}=0.000)$ and subjects with $\operatorname{LBP}(\mathrm{P}=0.000)$ (Table 2, Figure 2). Overall, the lumbar lordosis was significantly greater in the PLKF compared to prone-relaxed position in both subjects with and without LBP. The mean difference in lumbar lordosis between positions was 6.47 and 5.65 for subjects with LBP and without LBP respectively.

There was no statistically significant difference in the changes of lumbar lordosis after performing PLKF between subjects with and without LBP $(\mathrm{P}=0.65)$ (Table 3). However, the changes in lumbar lordosis were greater among patients with LBP compared to those without LBP.

\section{Discussion}

The current study shows changes in lumbar lordosis during active PLKF test in subjects with and without LBP. The results of this study demonstrated that there were statistically significant differences in lumbar lordosis between prone position and after PLKF in both subjects with and without LBP $(\mathrm{P}<0.0001)$. But the amount of changes in lordosis during PLKF test was not significant between two

Table 2 Lumbar lordosis in both groups

\begin{tabular}{llll}
\hline Variables & Before PLKF & After PLKF & P-value \\
\hline With no LBP & $35.02(9.25)$ & $40.67(13.09)$ & $\mathbf{0 . 0 0 0}$ \\
With LBP & $32.35(12.43)$ & $38.82(14.43)$ & $\mathbf{0 . 0 0 0}$ \\
\hline
\end{tabular}

Continuous data: Mean (Standard Deviation). Bold p-values indicate statistical significance.

LBP $=$ Low Back Pain, PLKF = Prone Lying Knee Flexion. 


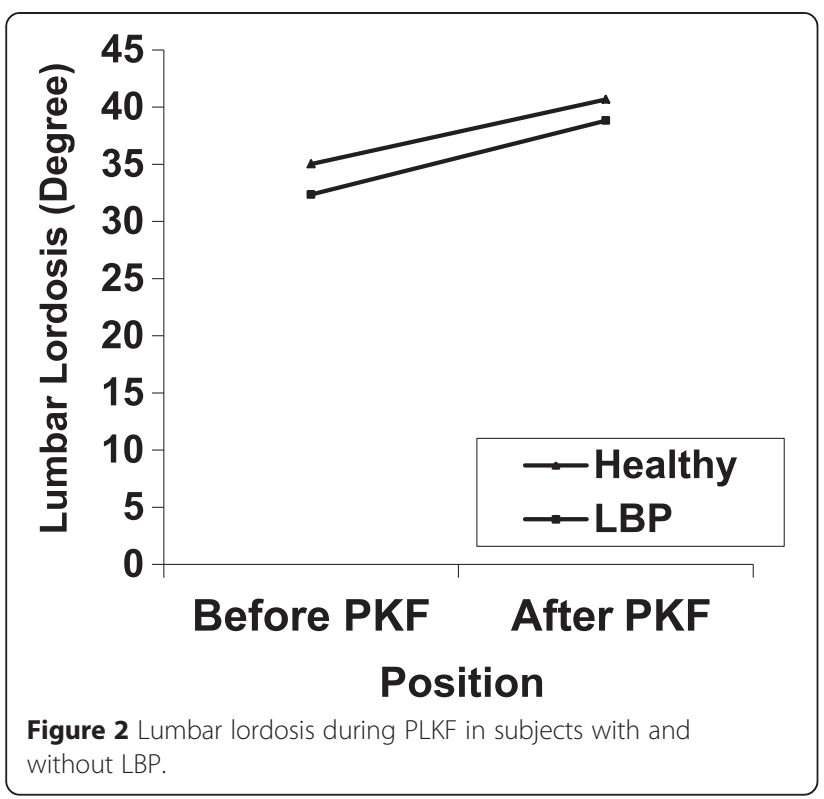

groups $(P=0.65)$. These changes were greater among patients with LBP compared to subjects without LBP.

In this study, the subjects had no pain during the test and none of the subjects reported that pain was a limiting factor to perform PLKF test, so, direct effects of pain on the measurement can be minimized.

Lumbar extension and anterior rotation of the pelvis are often observed during the PLKF test. Increase in the degree of lumbar lordosis during PLKF test found in both groups can be attributed to the accompanied lumbar extension during flexion of the knee. In theory, it is proposed that excessive anterior pelvic tilt, lumbar hyperextension and increased lumbar lordosis during the PLKF are commonly seen as abnormal movement patterns in patients with chronic LBP [5]. Investigators attributed these to muscle imbalance and altered activation of the lumbopelvic muscles [5].

Previous investigators attributed excessive lumbar extension and hyper lordosis during PLKF to a deficiency in controlling anterior pelvic rotation during PLKF because of muscular dysfunction in the lumbo-pelvic region [5,25]. Sahrmann [5] proposed the concept of "relative flexibility or stiffness" that has been linked to uncontrolled movement, pain and pathology by causing direction related stress and strain during various functional movements in the patients with LBP. Sahrmann

Table 3 Changes in lumbar lordosis between two groups

\begin{tabular}{llll}
\hline Variable & $\begin{array}{l}\text { With no LBP } \\
(\mathbf{n = 4 0 )}\end{array}$ & $\begin{array}{l}\text { With LBP } \\
(\mathbf{n = 4 0 )}\end{array}$ & P-value \\
\hline $\begin{array}{l}\text { Changes in lumbar lordosis } \\
\text { during PLKF }\end{array}$ & $5.65(10.13)$ & $6.47(6.67)$ & 0.65 \\
\hline
\end{tabular}

Continuous data: Mean (Standard Deviation).

$\mathrm{LBP}=$ Low Back Pain, PLKF = Prone Lying Knee Flexion.
[5] suggested that increased stiffness of the anterior supporting structures of the thigh, hip, knee and lumbar spine can result in compensatory exaggerated anterior pelvic tilt with lumbar extension motion during prone knee flexion or hip extension. In this study, stiffness in thigh and anterior supporting structures of the lumbar spine was not measured, just measured the change in lumbar lordosis during PLKF.

Scholtes et al. [26] found that during knee flexion and hip lateral rotation in prone lying, subjects with LBP demonstrated a greater maximal lumbar-pelvic rotation angle compared to those without LBP, as the lumbarpelvic region may move more frequently during the early ranges of lower limb movement in daily activities.

In this study, lumbar lordosis was significantly higher during PLKF compared to prone relaxed position in both subjects with and without LBP. However, this change in lumbar lordosis during PLKF was not significant between two groups. The reason for this may be due to the healthy subjects being recruited from university students and staff used to performing sustained postures and repeated movements in their daily activities.

It has been thought that, if the lumbar-pelvic motion occurs more during a limb movement, then the frequency of lumbar-pelvic motion may be increased through the day. The increased frequency of the movements in lumbarpelvic region can contribute to increased mechanical stress and strain on lumbar-pelvic region. This can also change the characteristics of muscular tissue, in turn, leading to abnormal movement patterns in lumbar-pelvic region [26,27]. Previous studies supported increased mobility of the lumbar-pelvic region in LBP patients which can be associated with degeneration of lumbar-pelvic region tissues $[28,29]$.

In this study, compensatory lumbar extension motion during active PLKF test may be due to instability in lumbar-pelvic region and also, excessive flexibility of movement of the lumbar spine in the direction of extension. This hypothesis has been supported by findings which suggest active limb movements which contribute to accumulation of tissue stress can affect decrease in spinal stability in patients with LBP [30]. However, more studies are needed to resolve the existing ambiguities in this field.

\section{Limitations}

We acknowledge some limitations. In this study the patients with chronic non-specific LBP were examined and other LBP patients (acute or specific LBP) were not examined. Another limitation of this study was that LBP subjects were not categorized based on movement system impairment-based categories for LBP as described by Sahrmann [5]. 
It has been suggested investigating the lumbar lordosis change in LBP patients with different movement system impairment-based categories. Again in this study, we did not measure lumbar -pelvic kinematics and electromyography (EMG) activity of the stabilizing and prime mover muscles during PLKF to find the pattern of muscles recruitment.

The fact that the healthy subjects were recruited from university students and staff performing sustained postures and repeated movements in their daily activities may be used to question the results showing no significant difference in lumbar lordosis change during PLKF between two groups.

Considering the non statistically different but measurable changes in lumbar lodosis during PLKF between subjects with and without LBP, we suggest that PLKF can be used as an evaluation tool of lumbar-pelvic movement patterns in the individuals with LBP and even healthy individuals with poor postural alignment and poor movement habits.

\section{Conclusion}

This study investigated the chansge in lumbar lordosis during PLKF test between subjects with and without LBP. The results of this study indicate an increase in the degree of lumbar lordosis during PLKF compared to prone-relaxed position in subjects with and without LBP. However, greater change in lumbar lordosis was found in the subjects with LBP compared to healthy subjects. More studies are needed to resolve the existing ambiguities in this field.

\section{Competing interests}

The authors declare that they have no competing interests.

\section{Authors' contributions}

AMA contributed to conception, design, analysis, interpretation of data and drafting the manuscript. AT carried out the data collection and drafting the manuscript. NK participated in design and interpretation of data. FE participated in analysis and helped to draft the manuscript. All authors read and approved the final manuscript.

\section{Author details \\ ${ }^{1}$ Department of Physical Therapy, University of Social Welfare and Rehabilitation Sciences, Velenjak, Tehran, Iran. ${ }^{2}$ University of Social Welfare and Rehabilitation Sciences, Velenjak, Tehran, Iran.}

Received: 25 July 2014 Accepted: 13 April 2015

Published online: 01 June 2015

\section{References}

1. Marras WS, Allread WG, Burr DL, Fathallah FA. Prospective validation of a low-back disorder risk model and an assessment of ergonomic interventions associated with manual materials handling tasks. Ergonomics. 2000;43:1866-86.

2. Lahiri S, Markkanen P, Levenstein C. The cost-effectiveness of occupational health interventions: preventing occupational back pain. Am J Ind Med. 2005;48(6):515-29.

3. Verhaak PF, Kerssens JJ, Dekker J, Sorbi MJ, Bensing JM. Prevalence of chronic benign pain disorder among adults: a review of the literature. Pain. 1998;77(3):231-9.
4. Gilgil E, Kacar C, Butun B, Tuncer T, Urhan S, Yildirim C, et al. Prevalence of low back pain in a developing urban setting. Spine. 2005;30:1093-8.

5. Sahrmann S. Diagnosis and treatment of movement impairment syndromes. 1. Missouri: Mosby. Inc; 2002. p. 121-92.

6. Jull GA, Janda V. Muscles and motor control in low back pain: Assessment and management. In: Twomey LT, Taylor JR, editors. Physical Therapy of the Low Back. Chuchill Livingstone: New York; 1987. p. 253-78.

7. Norris CM. Spinal stabilisation: an exercise programme to enhance lumbar stabilistion. Phys Ther. 1995;81(3):13-39.

8. Cholewicki J, Van Dieen JH, Arsenault AB. Muscle function and dysfunction in the spine. J Electromyoger Kinesiol. 2003;13(4):303-4.

9. Janda V. On the concept of postural muscles and posture in man. Aust J Physiother. 1983;29:83-4.

10. Janda V. Pain in the locomotor system-A broad approach. Aspects of manipulative therapy. Melbourne: Churchill Livingstone; 1985. p. 148-51.

11. O'Sullivan P, Phyty D, Twomey L, Allison GT. Evaluation of specific stabilizing exercise in the treatment of chronic low back pain with radiologic diagnosis of spondylolysis or spondylolisthesis. Spine. 1997;22(24):2959-67.

12. Hodges P, Moseley G. Pain and motor control of the lumbopelvic region: effect and possible mechanisms. J Electromyogr Kinesiol. 2003;13:361-70.

13. Hungerford B, Gilleard W, Hodges P. Evidence of altered lumbopelvic muscle recruitment in the presence of sacroiliac joint pain. Spine. 2003;28:1593-600.

14. Leinonen V, Kankaanpaa M, Airaksinen O, Hanninen O. Back and hip extensor activities during trunk flexion/extension: Effects of low back pain and rehabilitation. Arch Phys Med Rehabil. 2000;81:32-7.

15. Newcomer K, Jacobson T, Gabriel D, Larson DR, Brey RH, An KN. Muscle activation patterns in subjects with and without low back pain. Arch Phys Med Rehabil. 2002:83:816-21.

16. Nourbakhsh MR, Arab AM. Relationship between mechanical factors and incidence of low back pain. J Orthop Sports PhysTher. 2002;32:447-60.

17. Link CN, Nicholson GG, Shaddeau SA, Birch R, Gossman MR. Lumbar curvature in standing and sitting in two types of chairs: relationship of hamstring and hip flexor muscle length. PhysTher. 1990;70:611-8.

18. Nourbakhsh MR, Arab AM, Salavati M. The relationship between pelvic cross syndrome and chronic low back pain. J Back Musculoskeletal Rehabil. 2006;19:119-28.

19. Youdas JW, Garrett TR, Harmsen SS. Lumbar lordosis and pelvic inclination of asymptomatic adults. Phys Ther. 1996;76:1066-81.

20. Youdas JW, Garrett TR, Egan KS, Therneau TM. Lumbar lordosis and pelvic inclination in adults with chronic low back pain. Phys Ther. 2000;80:261-75.

21. Seidi F, Rajabi R, Ebrahimi TJ, Tavanai AR, Moussavi SJ. The Iranian flexible ruler reliability and validity in lumbar lordosis measurments. Sport Sci. 2009;2(2):95-9.

22. Hart DL, Rose SJ. Reliability of a noninvasive method for measuring the lumbar curve. Orthop Sports Phys Ther. 1986;8(4):180-4.

23. Youdas JW, Vj S, Garrett TR. Reliability of measurments of lumbar spine sagittal mobility obtained with the flexible curve. Orthop Sports Phys Ther. 1995;21(1):13-20.

24. Nourbakhsh MR, Moussavi SJ, Salavati M. Effects of lifestyle and work-related physical activity on the degree of lumbar lordosis and chronic low back pain in a Middle East population. Spinal Disord. 2001;14:283-92.

25. Jae-Seop O. Effects of Peforming an abdominal drwing-in maneuver during prone hip extension exercises on hip and back extensormuscle activity and amount of anterior pelvic tilt. J OrthopSports Phys Ther. 2007;37(6):320-4.

26. Scholtes SA, Gombatto SP, Van Dillen LR. Differences in lumbopelvic motion between people with and people without low back pain during two lower limb movement tests. Clin Biomech. 2009;24(1):7-12.

27. Van Dillen LR, Sahrmann SA, Norton BJ, Caldwell CA, Fleming D, McDonnell $M K$, et al. Effect of active limb movements on symptoms in patients with low back pain. J Orthop Sports Phys Ther. 2001;31:402-18.

28. Leone A, Guglielmi G, Cassar-Pullicino VN, Bonomo L. Lumbar intervertebral instability: a review. Radiology. 2007;245(1):62-77.

29. Singer KP, Fitzgerald D, Milne N. Neck retraction exercises and cervical disk disease. In: Singer KP, editor. Proceeding of the Biennial manipulative physiotherapist conference. Australia: Perth; 1993. p. 88-93.

30. Scholtes SA, Van Dillen LR. Gender-related differences in prevalence of lumbopelvic region movement impairments in people with low back pain. J Orthop Sports Phys Ther. 2007;37:744-53. 